\title{
A Novel Prognostic Model Based On Cytosolic DNA Sensing-Related Gene Signature for Pancreatic Cancer
}

\section{Chuan-Qi Xu}

People's hospital of Jingjiang

Kui-Sheng Yang

People's hospital of Jingjiang

Shu-Xian Zhao

People's Hospital of Jingjiang

Jian Lv (Dyks050605@163.com )

People's Hospital of Jingjiang

\section{Research Article}

Keywords: Pancreatic cancer, Cytosolic DNA sensing, Tumor immune, Prognosis model

Posted Date: September 16th, 2021

DOI: https://doi.org/10.21203/rs.3.rs-877931/v1

License: (c) (i) This work is licensed under a Creative Commons Attribution 4.0 International License. Read Full License 


\section{Abstract}

Objective: Pancreatic cancer (PC) is one of the most malignant tumors. Cytosolic DNA sensing have been found to play an essential role in tumor. In this study, a cytosolic DNA sensing-related genes (CDSRGs) signature was constructed and the potential mechanisms also been discussed.

Methods: The RNA expression and clinical data of PC were obtained from The Cancer Genome Atlas (TCGA) and Gene Expression Omnibus (GEO). Subsequently, univariate (UCR) and multivariate Cox regression (MCR) analyses were conducted to establish a prognostic model in the TCGA patients, which was verified by GEO patients. Cancer immune infiltrates were investigated via single sample gene set enrichment analysis (SSGSEA) and Tumor Immune Estimation Resource (TIMER). Finally, Gene Set Enrichment Analysis (GSEA) was used to investigate the related signaling pathways.

Results: A prognostic model comprising four genes (POLR2E,IL18, MAVS, and FADD) was established. The survival rate of patients in the low-risk group was significantly higher than that of patients in the high-risk group. In addition, CDSRGs-risk score was proved as an independent prognostic factor in PC. Immune infiltrates and drug sensitivity are associated with POLR2E,IL18, MAVS, and FADD expression.

Conclusions: In summary, we present and validated a CDSRGs risk model that is an independent prognostic factor and indicates the immune characteristics of PC. This prognostic model may facilitate the personalized treatment and monitoring.

\section{Introduction}

Pancreatic cancer (PC) is a common malignant tumor of the digestive tract. In 2020, there were approximately 496,000 new cases and 466,000 deaths worldwide[1]. The prevalence and incidence have increased in the past few years and continue to rise [2]. Despite the use of innovative therapeutic strategies for PC with a 5-year survival rate of $9 \%[3]$. Therefore, novel diagnostic biomarkers and effective therapeutic strategies are thus urgently required to improve the survival of patients with PC.

Cytosolic DNA is fundamental for mammalian organisms to control malignant transformation and tumor progression. DNA sensing has crucial functions in activate innate immune responses $[4,5]$. In response to cytosolic DNA, TANK-binding kinase 1(TBK1) and Interferon Regulatory Factor 3 (IRF3) mediating type I Interferon (IFN) induction[6]. Furthermore, type I IFN increased after chemotherapy, which can facilitate cross-priming and recruit T cells through the C-X-X motif chemokine 10 (CXCL10) pathway[7]. The cytosolic DNA sensors (primary cGAS and others) enhanced the tumor immune surveillance via activation with antigen-presenting cells leads to production of Tap2 and MHC-I [8]. The antitumor response of NK cells is primarily based on the cytosolic DNA sensing pathway, and identifies tumorderived cGAMP as a significant factor of tumor immunogenicity with inferences for cancer immunotherapy[9]. Therefore, cytosolic DNA sensing genes (CDSGs) is a very important factor affecting the prognosis for PC patients, but there has been little systematic study of CDSGs in PC. 
In this study, we downloaded original data from the TCGA and GEO database. Then, we established a prognostic signature of cytosolic DNA sensing-related genes using TCGA database and validated it in GEO database. Besides this, we further investigated the link between between cytosolic DNA sensing and tumor immune. This prognostic model may facilitate the personalized treatment and monitoring for patients with PC.

\section{Materials And Methods}

\section{Collection of genomic data}

We downloaded the TCGA (TCGA-PAAD) data, which includes expression data and clinical data, and mutation data (https://portal.gdc.cancer.gov/), which contained 172 PC samples. In addition, 63 PC patients from GSE57495 datasets were retrieved (https://www.ncbi.nlm.nih.gov/geo/). Pathological and clinical information is shown in Table 1.74 cytosolic DNA sensing genes were retrieved from PathCards (https://pathcards.genecards.org/)[10] and GSEA/MSigDB (http://www.gsea-msigdb.org/gsea/login.jsp) datasets (Supplementary Table1).

\section{Prognosis model construction}

Statistically significant CDSGs in the univariable Cox model were included in the multivariable Cox model. This model estimates the risk score were computed based on the following formula: Risk score $=\Sigma$ expression level of genei * $\beta$ i. $\beta$ represents the regression coefficient of genei. Then, patients were stratified into low-/high-risk groups using the median risk score as the cutoff value. Receiver operating characteristic curves (ROCs) drawn using ROC package in R. Area under the curve (AUC) was then determined. With the same statistical Analysis of the CDSGs signature was validated in the GEO cohort.

\section{Univariate (UCR) and multivariate cox regression (MCR) Analyses}

The correlation between patient survival and clinical factors was determined by UCR analyses in two sets. Multivariate Cox proportional hazards model was used to identify independent prognostic factors of survival.

\section{Principal components analysis, GSEA analysis, and nomogram generation}

We used the principal component analysis "PCA" package to separate samples based on the expression of signature genes. Pathway enrichment analysis was performed using GSEA (GSEA4.0.3). The "rms" package of $\mathrm{R}$ was used to generate the nomogram, which contained risk scores and clinical factors.

\section{Gene expression, mutation, and immune analysis in PC}

The GEPIA was also used to generate an expression difference value of signature genes (http://gepia.cancer-pku.cn/). We further verified the protein expression level of signature genes based on The Human Protein Atlas (HPA) database (https://www.proteinatlas.org/). The infiltration of immune cell 
types were quantified by ssGSEA method via "gsva" package. Boxplots were drawn using the R package ggplot. Summarize, analyze, and visualize mutation annotation format (MAF) files were using package "maftools" in PC. The copy number alternations (CNAs) of the signature genes on immune cell and the association between gene expression and immune infiltration was evaluated by applying the TIMER (http://timer.cistrome.org).

\section{Drug sensitivity analysis}

The drug sensitivity was downloaded from the CellMiner database (https://discover.nci.nih.gov/cellminer/)[11]. R package "impute", "limma”, "ggplot2", and "ggpubr" were used for the data processing, statistical analysis, and result visualization.

\section{Statistical analysis}

Statistical analyses were conducted using R version 4.0.4, SPSS 24.0 (IBM, NY, USA). Comparisons between groups were performed using the t-test or rank-sum test. The survival curves were constructed by K-M method. For linear correlation, the Pearson correlation coefficient was evaluated. P-values $<0.05$ were considered statistically significant.

\section{Result}

\section{Construction the CSRGs Signature of PC}

There were only four normal samples in TCGA dataset. Therefore, the differentially expressed CSRGs were did not obtained. First, the UCR analysis was performed. A total of 9 CSRGs, namely ZBP1, POLR2E, CASP8, IL18, MAVS, POLR3F, CASP10, POLR3H, and FADD, had a conspicuous connection with the overall survival (OS) rate (Fig. 1a, Table $2 \mathrm{P}<0.05$ ). Then, the MCR analysis revealed that four CSRGs, namely POLR2E, IL18, MAVS, and FADD, can act as independent prognostic factors for PC (Fig. 1b, $P<0.05)$. Above 4 genes were chosen to establish the prognostic model. The risk scores of samples in each dataset were calculated using the following formula. Risk score $=-0.8350$ * expression quantity of POLR2E +0.3468 * expression quantity of IL $18+(-0.5941)$ * expression quantity of MAVS +0.5718 * expression quantity of FADD (Table 3 ).

\section{Validation of prognostic CSRGs signature}

To confirm the reliability of the CSRGs signature developed in the TCGA samples. In both the TCGA and GEO cohorts, using a median cut-off risk score, the patients were divided into high- and low-risk groups. We found that patients with a high-risk score were correlated with worse outcomes in the TCGA samples, which was further validated from the GEO samples (Fig. 2a, f). The mortality rate in high-risk group was higher than low-risk group (Fig. 2b, g, d, i). Besides, the expression of IL18 and FADD was significantly increased in the high-risk group. The high-risk group had significantly lower POLR2E and MAVS levels than the low-risk group (Fig. 2c, h). PCA patients in low- and high-risk groups were separated into two different directions (Fig. 2e, j). 


\section{Independent prognostic value and construction of a nomogram of the risk signature.}

Both UCR and MCR analysis showed that the risk score was the independent prognostic factors for OS of

PC in the TCGA cohort (Fig. 3a, c) and GEO cohort (Fig 3b, d). The nomogram was constructed from the clinicopathological data as well as the developed prognostic model to predict the 1-,3-, and 5-year OS of PC (Fig. 4a, b). Time-dependent ROC curves also revealed the satisfactory predictive potential of the model (Fig. 4c, d). The above results indicated demonstrated the robustness of prognostic value of the four CSRGs in PC patients.

\section{Landscape of mutation profiles in two risk groups}

We found most of the gene mutations were missense mutations. The most common type of variant was a single nucleotide polymorphism, with $\mathrm{C}>\mathrm{T}$ (Fig. 5a, b). The top 30 most frequently mutated genes in two risk groups with ranked percentages (Fig. 5c, d). Besides, the frequencies of gene mutation of highrisk group (95.12\%) (Fig. 5c) were higher than low-risk group (87.5\%) (Fig. 5d), suggesting somatic mutation was positively correlated risk scores.

\section{Relationships between the signature and immune microenvironment}

Considering cytosolic DNA sensing was related to immune status, we further explored the correlations between risk scores and immune status. We found that the contents of most immune cells in high-risk group, including APC co-stimulation, iDCs, MHC class I, Neutrophils, and IFN Response Treg, NK cells and Tfh were significantly lower than those in low-risk group (Fig. 6c, d). Due to cytosolic DNA Sensing induced the productions of cytokines and chemokines. Therefore, the cytokines and chemokines also been discussed in two datasets. The results showed that the highly expressions of IL18, CSF2, and IL1RN in the high-risk group (Fig. 6a, b). On contrary, the low expression of CXCL12 in the low-risk group. Our result indicated that the CSRGs signature might relate to tumor immune and the production of cytokines and chemokines.

\section{Landscape of genetic variation of four signature genes in PC}

The investigation of CNV alteration frequency showed a prevalent CNV alteration in four signature genes, while POLR2E and MAVS had a widespread frequency of CNV deletion (Fig. 7a). The location of CNV alteration of four signature genes on chromosomes was shown in Fig. 7b. GEPIA were applied to validate the differentially expression levels of 4 signature genes. The results show that POLR2E, IL18, and FADD remarkably overexpressed in PC samples (Fig. 7c). The expression of MAVS is no significant difference (Fig. 7c). In addition, we performed protein expression analyses using the HPA database. The results indicated that IL18 and FADD were higher expression in PC tumor tissues than normal tissues. POLR2E and MAVS were lower expression in tumor tissues than normal tissues (Fig. 7d).

Four signature genes associated with immune infiltration 
We evaluated the relationship between four signature genes expression and immune status in TIMER. Positive correlation was observed between POLR2E expression and the infiltration of CD4+ T cells (Cor= $0.26 \mathrm{P}=6.39 \mathrm{e}-04)(\mathrm{Fig} .8 \mathrm{a})$. The results also suggested the infiltration of $\mathrm{B}$ cells (Cor $=0.335, \mathrm{P}=$ 7.70e-06), CD8+ T cells (Cor=0.394, $P=9.71 e-08)$, macrophages (Cor=0.529, $P=1.08 \mathrm{e}-13), D C$ (Cor=0.362, $P=1.16 \mathrm{e}-06)$ and neutrophils (Cor $=0.25, P=9.87 \mathrm{e}-04)$ positively correlated with MAVS expression (Fig. 8b). FADD expression was positively related to the infiltration of $B$ Cell (Cor $=0.201, P=$ 8.25e-03), CD8+ T (Cor=0.16, $P=3.60 \mathrm{e}-02)$, and DC (Cor=0.2, $P=8.56 \mathrm{e}-03)$ (Fig. 8c). IL18 expression was positively related to the $B$ cells (Cor $=0.165, P=3.12 e-02), C D 8+T$ cells (Cor $=0.215, P=4.71 e-03$ ), neutrophils (Cor=0.188, $P=1.36 \mathrm{e}-02)$, and $D C$ (Cor= 0.16, $P=3.71 \mathrm{e}-02)$ (Fig. 8d). The CNAs of the identified four signature genes, including arm-level, deletion, and arm-level gain (Fig. 9). These results demonstrated that four signature genes had pivotal regulatory effects on the tumor immune microenvironment (TIME) for PC patients.

\section{Four signature genes associated with drug sensitivity}

The CellMiner database was used to investigate the relationship between the drug sensitivity and the expression of four signature genes. The results illustrated that IL18 expression was associated with Paclitaxel (Cor $=-0.364, p=0.004)$, Afatinib (Cor $=0.293, p=0.023)$, Hypothemycin (Cor $=-0.354, p=0.006)$ drug sensitivity. POLR2E expression was associated with Gemcitabine (Cor=0.273, $p=0.035)$, Cisplatin (Cor= 0.297, $p=0.021$ ), Carboplatin (Cor $=0.262, p=0.043$ ) drug sensitivity. MAVS expression was associated with Oxaliplatin (Cor $=-0.334, p=0.009$ ), Rapamycin (Cor= 0.371, p=0.004), Paclitaxel (Cor= $-0.278, p=0.031$ ) drug sensitivity. FADD expression was associated with Vinorelbine (Cor $=-0.357$, $p=0.005)$, Fluorouracil (Cor $=-0.333, p=0.009)$, Tamoxifen (Cor $=-0.319, p=0.013)$ drug sensitivity (Fig 10).

\section{GSEA for identifying the cytosolic DNA sensing-related gene signature signaling pathways}

GSEA analysis was conducted to compare more specifically the significantly enriched pathways between two groups. Interferon-alpha response and Glycolysis pathway were highly enriched in high-risk group in both datasets (Fig. 11).

\section{Discussion}

Cytosolic DNA sensing is intimately connected to the secretion of cytokines that support antitumor immunity [12]. Multiple sensors of cytosolic DNA including CGAS, AIM2, IFI16, and STING, as well as their signal transducer TBK1, are expressed at lower levels in tumors $[13,14]$. Intratumoral injection of Vadimezan (the stimulator of STING) induces regression of established melanomas, colorectal carcinomas and mammary carcinomas, along with the activation of systemic immunity with antimetastatic effects[15]. Therefore, the strategies through activating DNA sensing pathways toward enhancing antitumor immunity in clinical studies.

In this study, a novel prognostic model of four cytosolic DNA sensing -related genes was constructed in this study, which could effectively predict the survival of PC patients. 
Furthermore, URC and MRC analysis results suggested the prognostic model as an independent predictor. A diagnostic nomogram was constructed to intuitively predict the OS, which be useful for both short and long term follow up.

Among the four genes in the prognostic model that we constructed, The POLR2E expression associate with poorer OS in acute myelocytic leukemia (AML) and prostate cancer risk [16, 17]. POLR2E increased expression is a radiation-sensitive factor, and it play essential roles in the proliferation and apoptosis of HCC cells [18]. However, POLR2E relate to the susceptibility and pathogenesis of PC has not been extensively examined. The mitochondrial antiviral signaling (MAVS) protein acts as an essential adaptor in the RIG-I-like receptor (RLR) signaling pathway, which plays an important role in host defenses against viral infections by inducing IFN-I production[19]. MAVS low expression was associated with a wide spectrum of malignant tumors [20]. Hence, MAVS activation may have tumor-suppressive effects in cancer cells. The Fas-associated death domain gene (FADD), a classical adaptor protein mediating apoptotic stimuli-induced cell death, protects pancreatic cancer cells from drug-induced apoptosis. Many studies revealed that FADD is overexpressed in many tumors, including PC [21, 22]. Lack of FADD contributes to immune escape and resistance to chemotherapy in AML cells [23]. In addition, FADD inhibited NF-KB activation in response to cytokine stimulation [24]. Interleukin-18 (IL-18) was originally identified as interferon (IFN)- $y$-inducing factor. IL-18 is significantly increased in the serum of patients with PC compared to healthy donors [25]. A higher free IL-18 serum concentration was detected in patients with locally advanced or metastatic PC [26]. IL18 promotes proliferation and invasion of PC cells via the NF-kB pathway. Furthermore, IL-18 derived from PC cells induced regulatory B cells (Breg) to promote immune tolerance [27]. Combination of the IL18 and NF-kB pathway inhibitor had an antitumor therapeutic effect on PC [28]. Our results also showed that four signature genes expression was associated with the immune infiltration and drug sensitivity, which was consistent with past results[29, 30].

Furthermore, our result indicated that the risk signature might associated with the production of cytokines and chemokines including IL18, CSF2, CXCL12, and IL1RN. Chemokines is critical in immune evasion[31]. For instance, indirect recruitment of tumor associated macrophage (TAMs) via CXCL12-induced IL-6 production leads to reinforces resistance to gemcitabine in PC cells [32]. The GSEA analysis also showed risk scores positively associated with interferon-alpha response, which is closely related to cytosolic DNA sensing and the production of chemokines [33]. Above results implicating that the risk signature might be involved in the generation of cytokines and chemokines via interferon-alpha response pathway, and finally affect the prognosis and immune status in PC patients.

In summary, we constructed and validated a novel cytosolic DNA sensing related prognostic signature which might be important clinically as an independent prognostic biomarker that can predict the prognosis, drug sensitivity, and reflecting the immune status. However, all the data analyzed were retrospective and from public datasets, and further research is still needed to verify our findings.

\section{Declarations}


Acknowledgements: None.

\section{Authors' contributions}

$\mathrm{KY}$ and CQ contributed to the conception and design of the study; CQ collected data and wrote the manuscript; SX performed the data analysis and constructed the figures and tables; CQ and JV reviewed and revised the manuscript and were involved in the conception of the study. Additionally, JV was responsible for the organization, revision, and submission of this manuscript. All authors read and approved the final manuscript.

\section{Availability of data and materials}

The datasets analyzed was acquired from The Cancer Genome Atlas (TCGA) database (https://portal.gdc.cancer.gov/) and GEO database (http://www.ncbi.nlm.nih.gov/geo/).

\section{Funding: None.}

\section{Ethics approval and consent to participate}

This was not applicable to this manuscript.

\section{Consent for publication}

Consent for publication was obtained from all participants.

\section{Competing interests}

The authors declare that they have no competing interests.

\section{References}

1. Sung H, Ferlay J, Siegel RL, Laversanne M, Soerjomataram I, Jemal A, Bray F: Global Cancer Statistics 2020: GLOBOCAN Estimates of Incidence and Mortality Worldwide for 36 Cancers in 185 Countries. CA Cancer J Clin 2021, 71:209-249.

2. Oshi M, Tokumaru Y, Patel A, Yan L, Matsuyama R, Endo I, Katz MHG, Takabe K: A Novel Four-Gene Score to Predict Pathologically Complete (R0) Resection and Survival in Pancreatic Cancer. Cancers (Basel) 2020, 12.

3. Sohal DPS, Willingham FF, Falconi M, Raphael KL, Crippa S: Pancreatic Adenocarcinoma: Improving Prevention and Survivorship. Am Soc Clin Oncol Educ Book 2017, 37:301-310. 
4. Ishikawa H, Barber GN: STING is an endoplasmic reticulum adaptor that facilitates innate immune signalling. Nature 2008, 455:674-678.

5. Deng L, Liang H, Xu M, Yang X, Burnette B, Arina A, Li X-D, Mauceri H, Beckett M, Darga T, et al: STING-Dependent Cytosolic DNA Sensing Promotes Radiation-Induced Type I Interferon-Dependent Antitumor Immunity in Immunogenic Tumors. Immunity 2014, 41:843-852.

6. Kwon J, Bakhoum SF: The Cytosolic DNA-Sensing cGAS-STING Pathway in Cancer. Cancer Discov 2020, 10:26-39.

7. Sistigu A, Yamazaki T, Vacchelli E, Chaba K, Enot DP, Adam J, Vitale I, Goubar A, Baracco EE, Remédios C, et al: Cancer cell-autonomous contribution of type I interferon signaling to the efficacy of chemotherapy. Nature Medicine 2014, 20:1301-1309.

8. Kato K, Omura H, Ishitani R, Nureki OJArob: Cyclic GMP-AMP as an Endogenous Second Messenger in Innate Immune Signaling by Cytosolic DNA. 2017, 86:541-566.

9. Yu L, Liu P: Cytosolic DNA sensing by cGAS: regulation, function, and human diseases. Signal Transduct Target Ther 2021, 6:170.

10. Belinky F, Nativ N, Stelzer G, Zimmerman S, Iny Stein T, Safran M, Lancet DJDtjobd, curation: PathCards: multi-source consolidation of human biological pathways. 2015, 2015.

11. Reinhold W, Sunshine M, Liu H, Varma S, Kohn K, Morris J, Doroshow J, Pommier YJCr: CellMiner: a web-based suite of genomic and pharmacologic tools to explore transcript and drug patterns in the $\mathrm{NCl}$ 60 cell line set. 2012, 72:3499-3511.

12. Vanpouille-Box C, Demaria S, Formenti S, Galluzzi LJCc: Cytosolic DNA Sensing in Organismal Tumor Control. 2018, 34:361-378.

13. Yang C, Huang H, Chang Y, Lin C, Lai I, Chang JJO: DNA-Sensing and Nuclease Gene Expressions as Markers for Colorectal Cancer Progression. 2017, 92:115-124.

14. Li C, Zhang Y, Cheng X, Yuan H, Zhu S, Liu J, Wen Q, Xie Y, Liu J, Kroemer G, et al: PINK1 and PARK2 Suppress Pancreatic Tumorigenesis through Control of Mitochondrial Iron-Mediated Immunometabolism. 2018, 46:441-455.e448.

15. Corrales L, Glickman L, McWhirter S, Kanne D, Sivick K, Katibah G, Woo S, Lemmens E, Banda T, Leong J, et al: Direct Activation of STING in the Tumor Microenvironment Leads to Potent and Systemic Tumor Regression and Immunity. 2015, 11:1018-1030.

16. Chen B, Wang S, Ma G, Han J, Zhang J, Gu X, Feng X: The association of POLR2E rs3787016 polymorphism and cancer risk: a Chinese case-control study and meta-analysis. Biosci Rep 2018, 38. 
17. Wei J, Xie Q, Liu X, Wan C, Wu W, Fang K, Yao Y, Cheng P, Deng D, Liu Z: Identification the prognostic value of glutathione peroxidases expression levels in acute myeloid leukemia. Ann Trans/ Med 2020, 8:678.

18. Yang $\mathrm{H}, \mathrm{Gu} J$, Zheng Q, Li M, Lian X, Miao J, Jiang J, Wei W: RPB5-mediating protein is required for the proliferation of hepatocellular carcinoma cells. J Biol Chem 2011, 286:11865-11874.

19. Biacchesi S, LeBerre M, Lamoureux A, Louise $Y$, Lauret E, Boudinot P, Brémont M: Mitochondrial antiviral signaling protein plays a major role in induction of the fish innate immune response against RNA and DNA viruses. J Virol 2009, 83:7815-7827.

20. Zhang W, Gong J, Yang H, Wan L, Peng Y, Wang X, Sun J, Li F, Geng Y, Li D, et al: The Mitochondrial Protein MAVS Stabilizes p53 to Suppress Tumorigenesis. 2020, 30:725-738.e724.

21. Zhang R, Liu Y, Hammache K, He L, Zhu B, Cheng W, Hua ZJOl: The role of FADD in pancreatic cancer cell proliferation and drug resistance. 2017, 13:1899-1904.

22. Pattje WJ, Melchers LJ, Slagter-Menkema L, Mastik MF, Schrijvers ML, Gibcus JH, Kluin PM, Hoegen-Chouvalova $\mathrm{O}$, van der Laan BF, Roodenburg $\mathrm{JL}$, et al: FADD expression is associated with regional and distant metastasis in squamous cell carcinoma of the head and neck. Histopathology 2013, 63:263-270.

23. Tourneur L, Buzyn A, Chiocchia GJMi: FADD adaptor in cancer. 2005, 4:1.

24. Chao W, Shen Y, Li L, Zhao H, Meiler SE, Cook SA, Rosenzweig A: Fas-associated death-domain protein inhibits TNF-alpha mediated NF-kappaB activation in cardiomyocytes. Am J Physiol Heart Circ Physiol 2005, 289:H2073-2080.

25. Bellone G, Smirne C, Mauri F, Tonel E, Carbone A, Buffolino A, Dughera L, Robecchi A, Pirisi M, Emanuelli GJCi, immunotherapy : Cll: Cytokine expression profile in human pancreatic carcinoma cells and in surgical specimens: implications for survival. 2006, 55:684-698.

26. Daley D, Mani V, Mohan N, Akkad N, Pandian G, Savadkar S, Lee K, Torres-Hernandez A, Aykut B, Diskin B, et al: NLRP3 signaling drives macrophage-induced adaptive immune suppression in pancreatic carcinoma. 2017, 214:1711-1724.

27. Zhao Y, Shen M, Feng Y, He R, Xu X, Xie Y, Shi X, Zhou M, Pan S, Wang M, et al: Regulatory B cells induced by pancreatic cancer cell-derived interleukin-18 promote immune tolerance via the PD-1/PD-L1 pathway. 2018, 9:14803-14814.

28. Guo X, Zheng L, Jiang J, Zhao Y, Wang X, Shen M, Zhu F, Tian R, Shi C, Xu M, et al: Blocking NF-kB Is Essential for the Immunotherapeutic Effect of Recombinant IL18 in Pancreatic Cancer. 2016, 22:59395950. 
29. Ibrahim S, Ringel J, Schmidt C, Ringel B, Müller P, Koczan D, Thiesen H, Löhr MJP: Pancreatic adenocarcinoma cell lines show variable susceptibility to TRAlL-mediated cell death. 2001, 23:72-79.

30. Gong WJ, Peng JB, Yin JY, Li XP, Zheng W, Xiao L, Tan LM, Xiao D, Chen YX, Li X, et al: Association between well-characterized lung cancer IncRNA polymorphisms and platinum-based chemotherapy toxicity in Chinese patients with lung cancer. Acta Pharmacol Sin 2017, 38:581-590.

31. Pancreatic Cancer-Associated Stellate Cells Promote Differentiation of Myeloid-Derived Suppressor Cells in a STAT3-Dependent Manner \%J Cancer Research. 2013, 73:3007-3018.

32. Zhang H, Wu H, Guan J, Wang L, Ren X, Shi X, Liang Z, Liu TJO: Paracrine SDF-1a signaling mediates the effects of PSCs on GEM chemoresistance through an IL- 6 autocrine loop in pancreatic cancer cells. 2015, 6 .

33. Keating SE, Baran M, Bowie AGJTil: Cytosolic DNA sensors regulating type I interferon induction. 2011, 32:574-581.

\section{Tables}

Table 1. The Clinical characteristics of the patients in TCGA and GEO datasets. 


\begin{tabular}{|c|c|c|c|}
\hline \multirow[t]{2}{*}{ Characteristics } & & \multicolumn{2}{|l|}{ Datasets } \\
\hline & & TCGA $(n=179)$ & GSE57495 $(n=63)$ \\
\hline \multirow[t]{2}{*}{ Outcome } & Alive & $86(48.04 \%)$ & $21(33.33 \%)$ \\
\hline & Dead & $93(51.96)$ & $42(66.67 \%)$ \\
\hline \multirow[t]{2}{*}{ Gender } & Female & $81(45.25 \%)$ & - \\
\hline & Male & $98(54.75 \%)$ & - \\
\hline \multirow[t]{5}{*}{ Grade } & Grade1 & $31(17.32 \%)$ & - \\
\hline & Grade2 & $95(53.07 \%)$ & - \\
\hline & Grade3 & $50(27.93 \%)$ & - \\
\hline & Grade4 & $1(0.56 \%)$ & - \\
\hline & NA & $2(1.12 \%)$ & - \\
\hline \multirow[t]{5}{*}{ Stage } & Stage1 & $20(11.17 \%)$ & $13(20.63 \%)$ \\
\hline & Stage2 & 147(82.12\%) & $50(79.37 \%)$ \\
\hline & Stage3 & $4(2.23 \%)$ & - \\
\hline & Stage4 & $5(2.79 \%)$ & - \\
\hline & NA & $3(1.68 \%)$ & - \\
\hline \multirow[t]{5}{*}{ Pathological T } & T1 & $6(3.35 \%)$ & - \\
\hline & $\mathrm{T} 2$ & $23(12.85 \%)$ & - \\
\hline & T3 & $144(80.45 \%)$ & - \\
\hline & T4 & $4(2.23 \%)$ & - \\
\hline & NA & $2(1.12 \%)$ & - \\
\hline \multirow[t]{3}{*}{ Pathological M } & MO & $81(45.25 \%)$ & - \\
\hline & M1 & $5(2.79 \%)$ & - \\
\hline & NA & $93(51.96 \%)$ & - \\
\hline \multirow[t]{3}{*}{ Pathological N } & NO & $48(26.82 \%)$ & - \\
\hline & $\mathrm{N} 1$ & 127(70.95\%) & - \\
\hline & NA & $4(2.23 \%)$ & - \\
\hline
\end{tabular}

NA = not applicable 
Table 2. A total of 9 cytosolic DNA sensing-related genes significantly associated with overall survival according to univariate Cox regression analysis.

\begin{tabular}{lllll} 
Id & HR & HR.95L & HR.95H & pvalue \\
\hline ZBP1 & 1.1380 & 1.0018 & 1.2926 & 0.0469 \\
\hline POLR2E & 0.6098 & 0.3969 & 0.9367 & 0.0239 \\
\hline CASP8 & 1.3708 & 1.0846 & 1.7326 & 0.0083 \\
\hline IL18 & 1.4079 & 1.1499 & 1.7239 & 0.0009 \\
\hline MAVS & 0.6442 & 0.4585 & 0.9052 & 0.0113 \\
\hline POLR3F & 0.6616 & 0.4595 & 0.9526 & 0.0263 \\
\hline CASP10 & 1.2802 & 1.0127 & 1.6185 & 0.0389 \\
\hline POLR3H & 0.6714 & 0.4793 & 0.9404 & 0.0205 \\
\hline FADD & 1.3515 & 1.0034 & 1.8203 & 0.0474
\end{tabular}

Table 3. Details of the 4 cytosolic DNA sensing-related genes significantly associated with overall survival used to build the risk model.

\begin{tabular}{llllll} 
Id & Coef & HR & HR.95L & HR.95H & P-value \\
\hline POLR2E & -0.8351 & 0.4338 & 0.2216 & 0.8494 & 0.0149 \\
\hline IL18 & 0.3468 & 1.4146 & 1.0249 & 1.9523 & 0.0349 \\
\hline MAVS & -0.5942 & 0.5520 & 0.3519 & 0.8659 & 0.0097 \\
\hline FADD & 0.5718 & 1.7715 & 0.9974 & 3.1463 & 0.0500
\end{tabular}

\section{Figures}

a

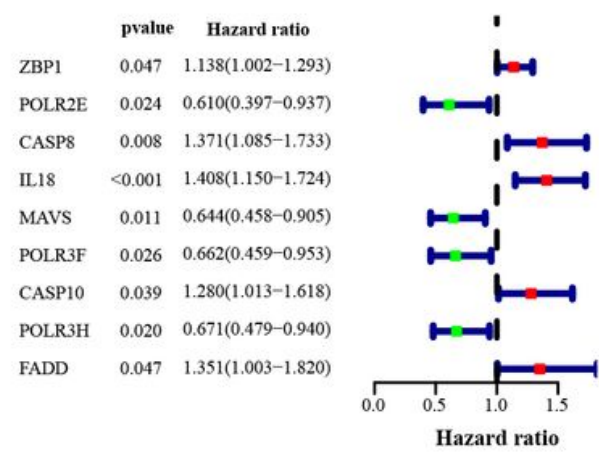

$\mathrm{b}$

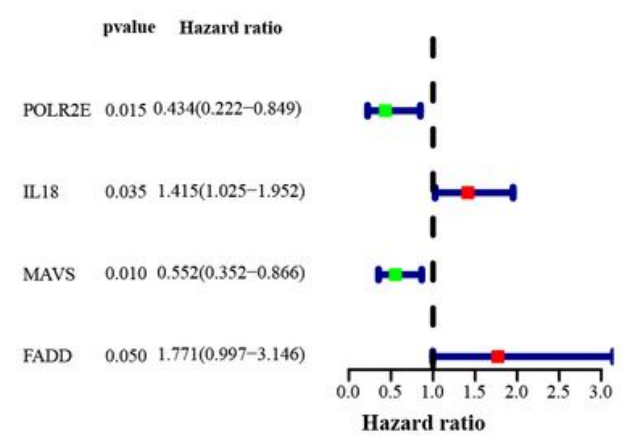


Figure 1

Construction of the cytosolic DNA sensing-Related gene signature. a Univariate Cox analysis (UCR) of the cytosolic DNA sensing-related genes. b Multivariate Cox analysis (MCR) results.
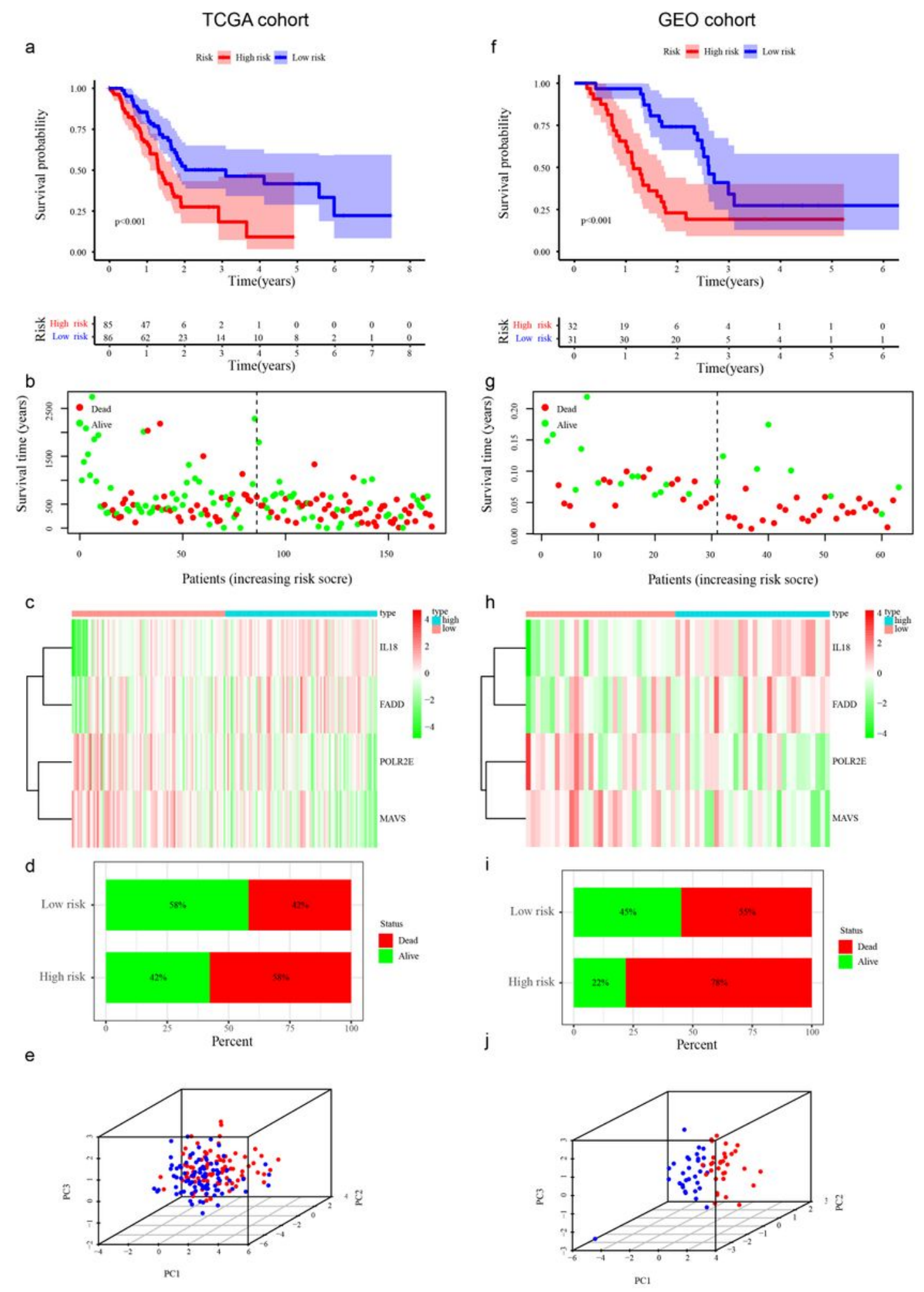

Figure 2 
Evaluation of Risk Score. a, $\mathrm{f} \mathrm{K}-\mathrm{M}$ curve in the TCGA and GEO groups. b, $\mathrm{g}$ The distribution of risk score in survival outcome in the TCGA and GEO groups. $\mathrm{c}, \mathrm{h}$ The expression pattern of four signature genes in the TCGA and GEO groups. $d$, i The mortality rate of patients in high-/low-risk groups in the TCGA and GEO groups. e, $j$ The principal component analysis (PCA) shows the distinguished distribution of high-/low-risk patients based on the risk model in the TCGA and GEO groups.

a

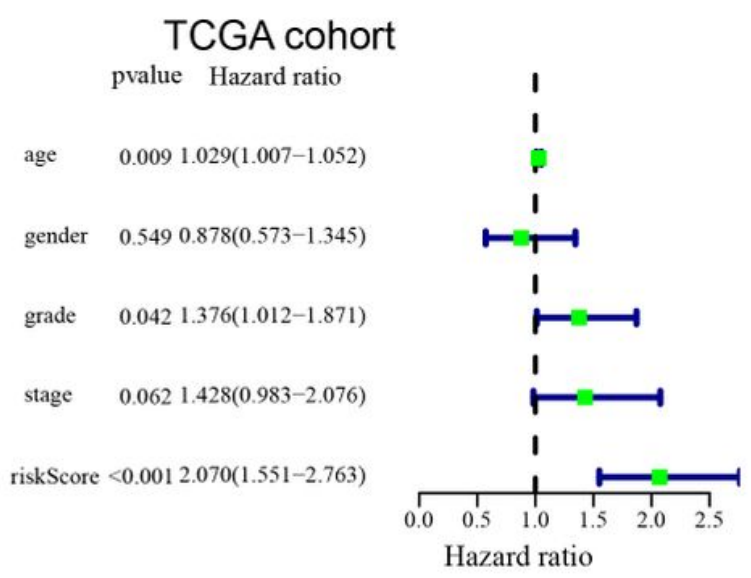

C

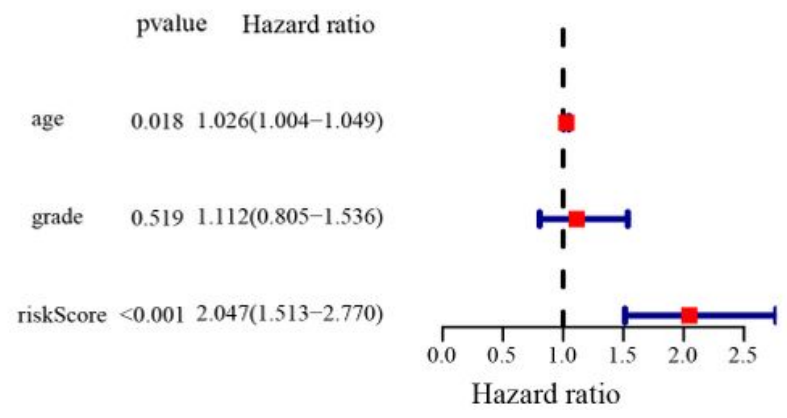

b

GEO cohort

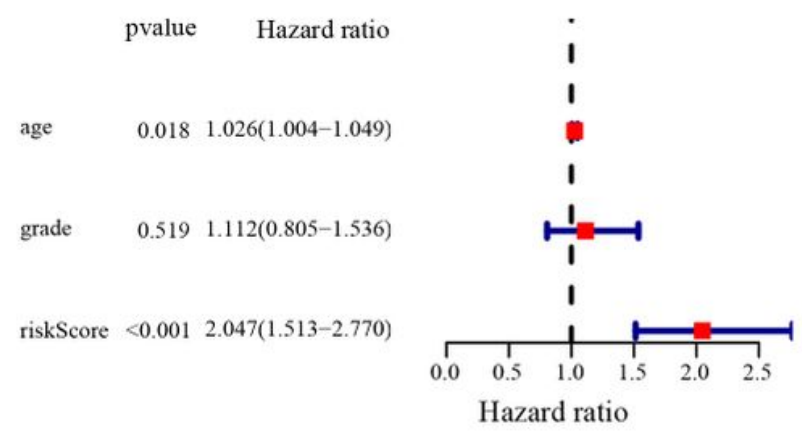

d

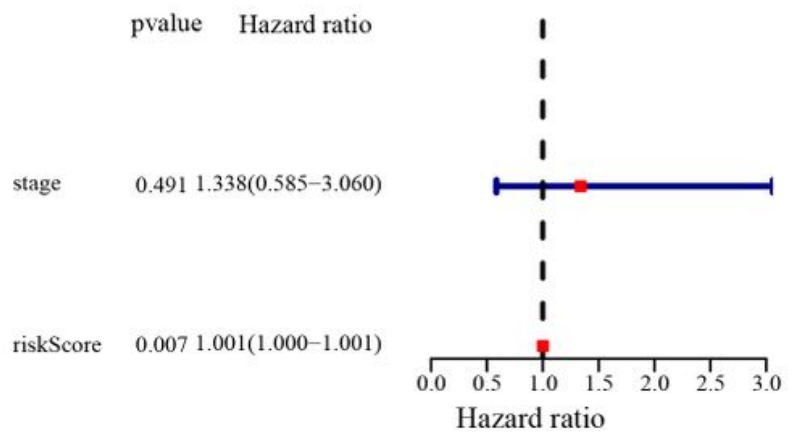

Figure 3

Independent predictive value of the risk signature. a-d Forest plot of the UCR and MCR analyses on clinical characteristics and risk score results in the TCGA and GEO cohort. 
TCGA cohort

a

Points

age

gender

grade

stage

riskScore

Total Points

1-year survival

2-year survival

3-year survival

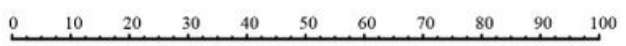

354045505560657075808590

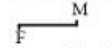

G1 $\stackrel{\text { Gं } 3}{G 4}$

stagel stage3 stage2
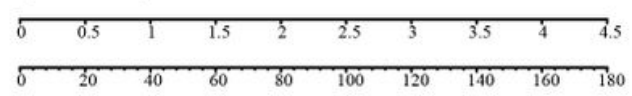

$\begin{array}{llllllllll}0.9 & 0.8 & 0.7 & 0.6 & 0.5 & 0.4 & 0.3 & 0.2 & 0.10 .05\end{array}$

$\begin{array}{lllllllllll}0.9 & 0.8 & 0.7 & 0.6 & 0.5 & 0.4 & 0.3 & 0.2 & 0.10 .05\end{array}$

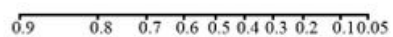

C

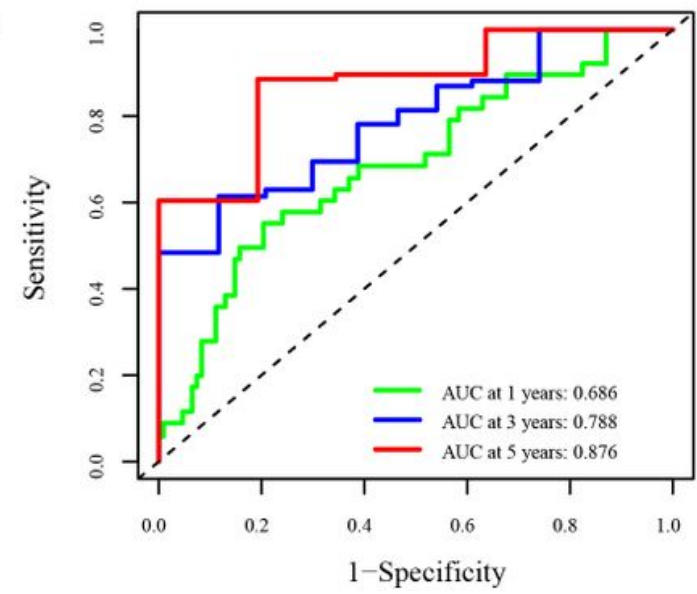

b

Points

stage

riskScore

Total Points

1-year survival

2-year survival

3-year survival
GEO cohort
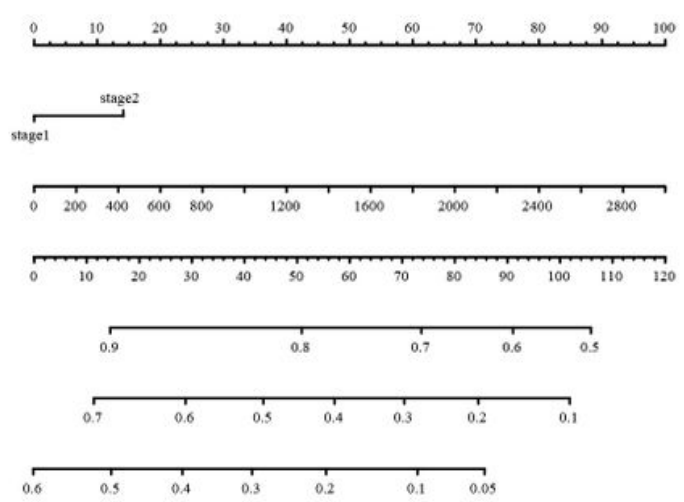

d

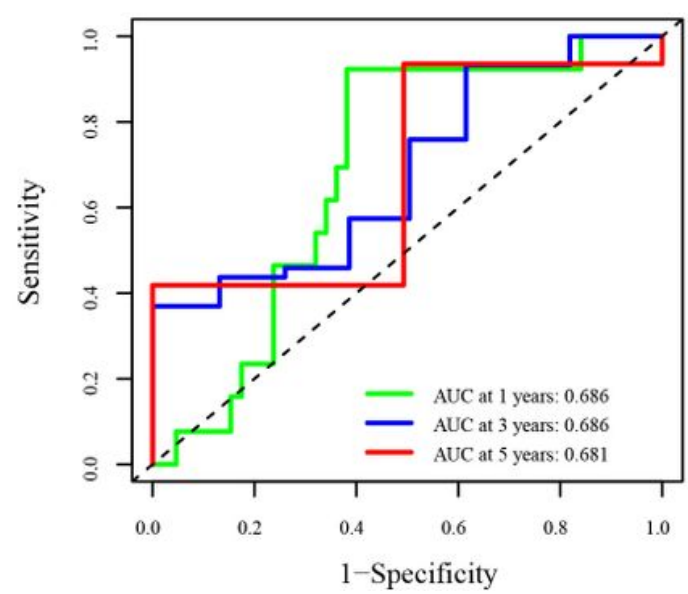

\section{Figure 4}

Nomogram for predicting OS based on clinical variables risk score. $a, b$ Construction of the nomogram in the TCGA and GEO cohort. c, d Time-dependent ROC analysis for 1-, 3-, and 5-year OS of a prognostic model in the TCGA and GEO cohort. 
a

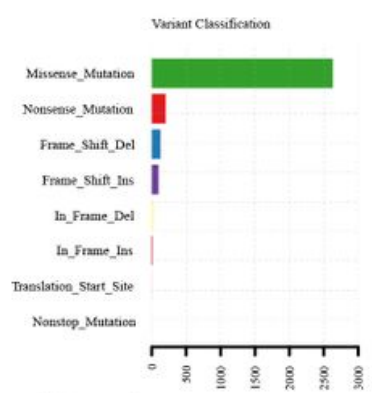

Vicivess per semple
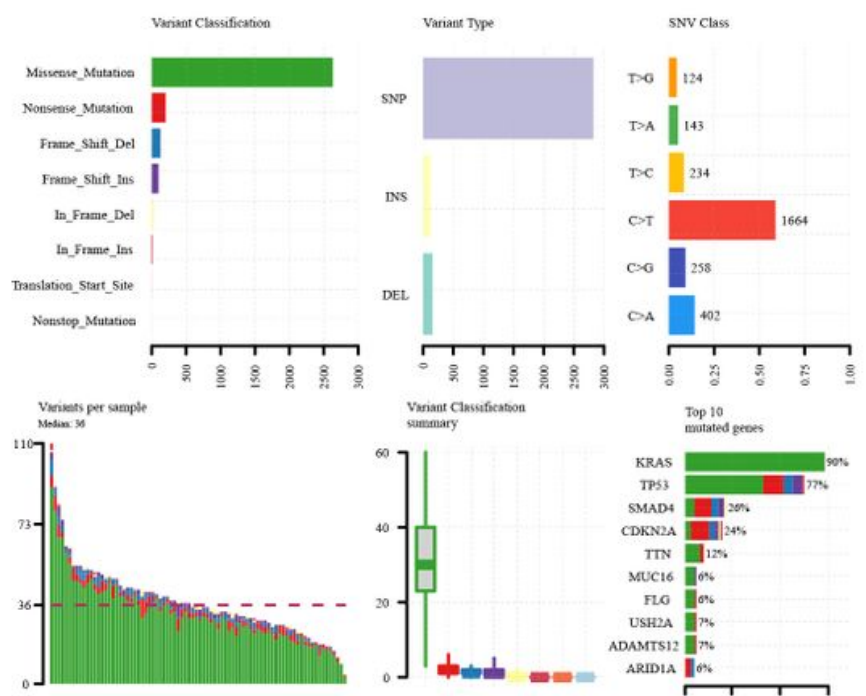

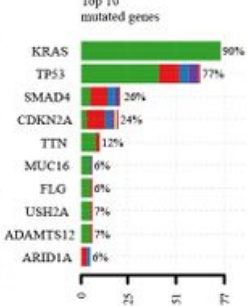

C

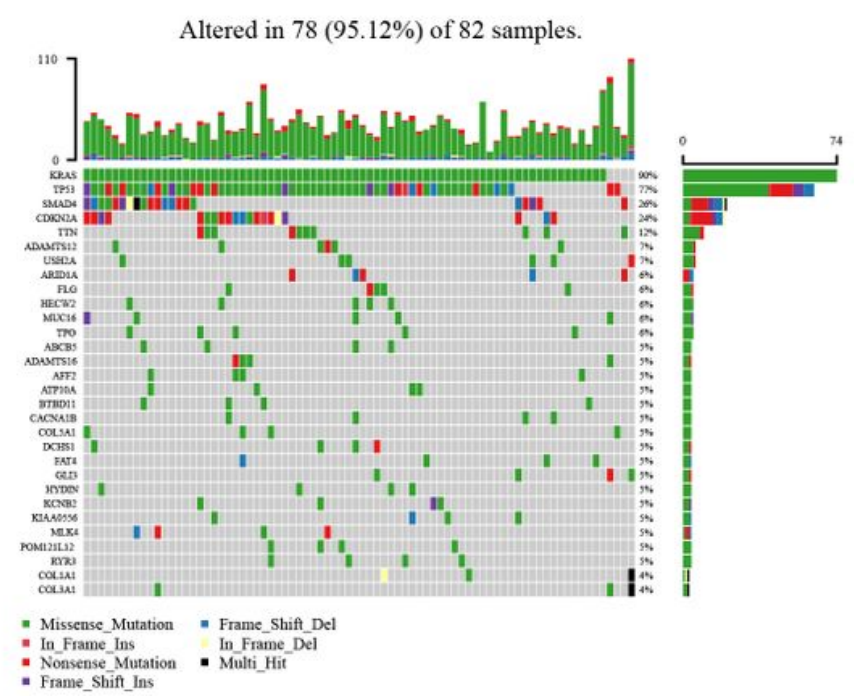

b
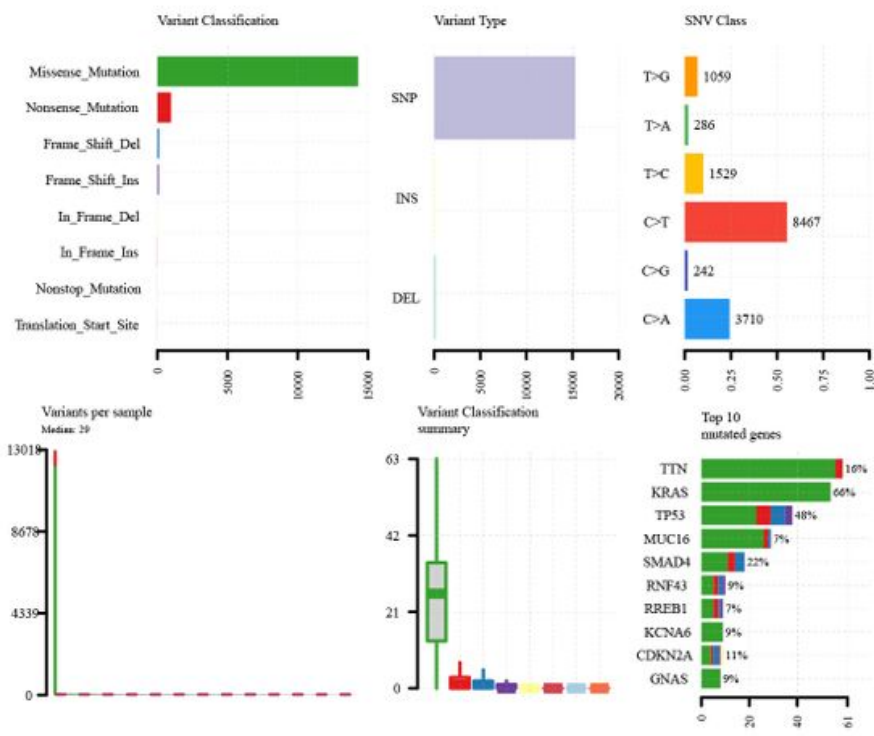

d

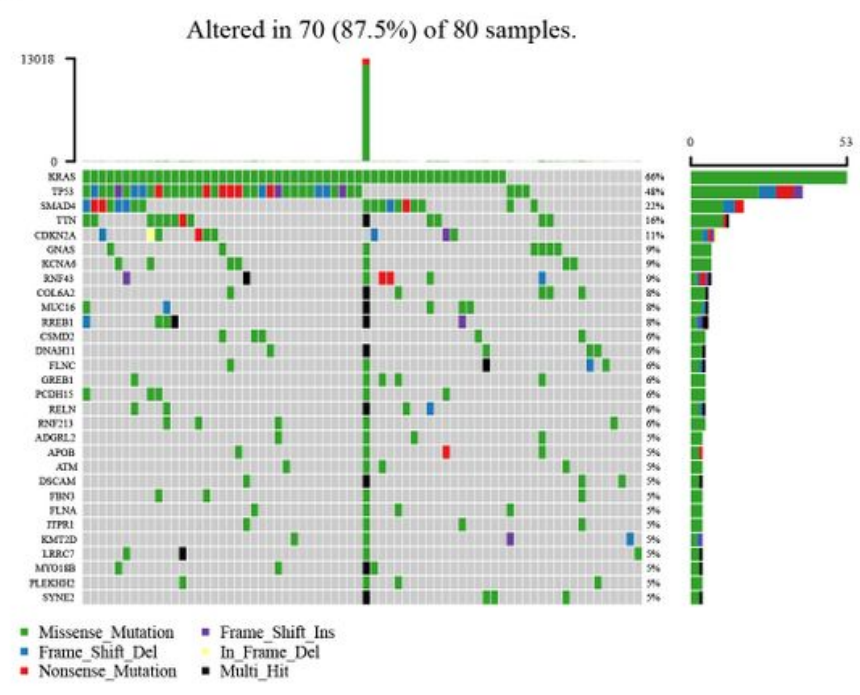

Figure 5

Landscape of mutation profiles in the high-/low-risk PC patients. a, b Overview of mutation types in the high-/low-risk group. c, d Waterfall Plot of the top 30 genes with the most mutations in the high-/low-risk group. 
a

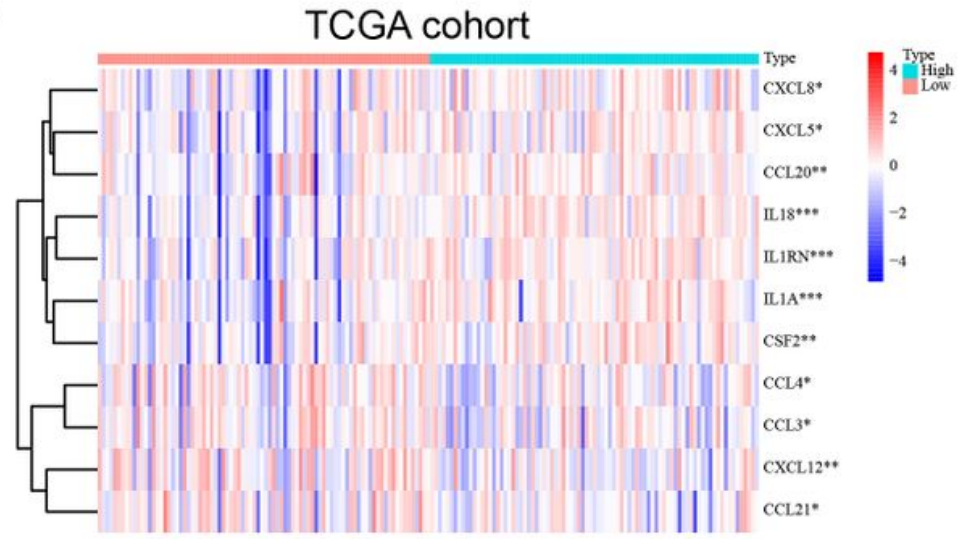

C

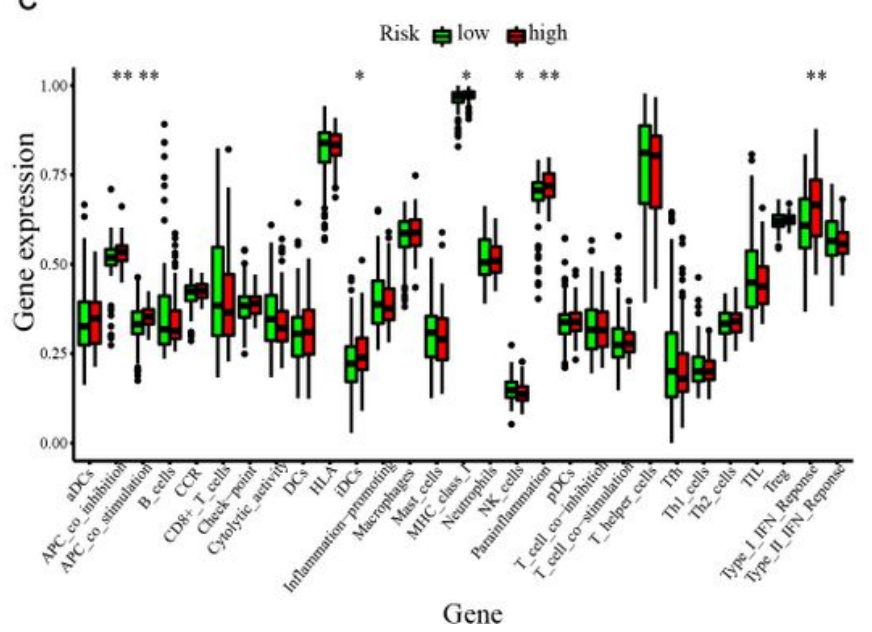

b
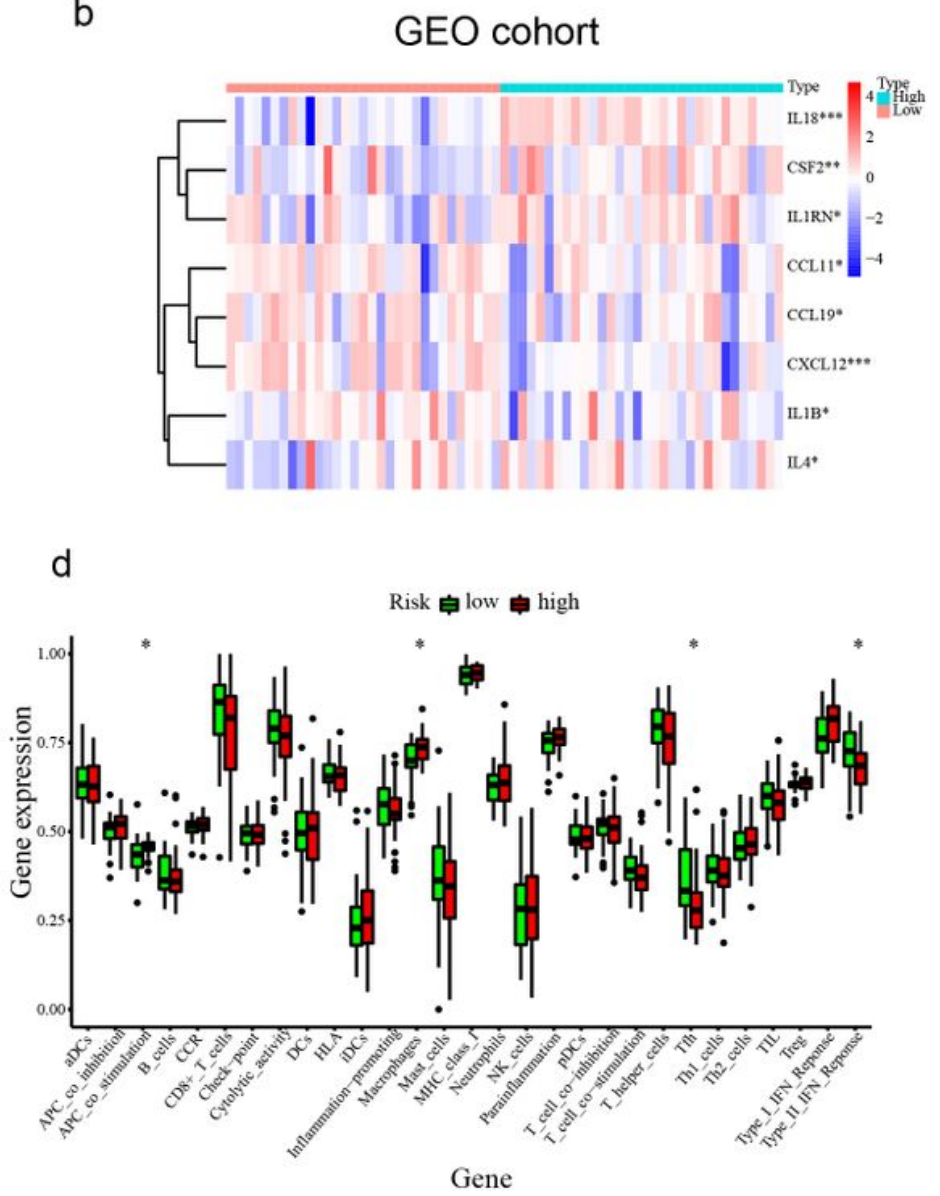

Figure 6

The differences of the sSGSEA scores and chemokines between two risk groups. $a, b$ The correlations between risk score and chemokines in the TCGA and GEO group. c, d The correlations between risk score and ssGSEA scores in the TCGA and GEO group. *P $<0.05, * \star P<0.01$, and ${ }^{\star * \star} P<0.005$. 
a

b
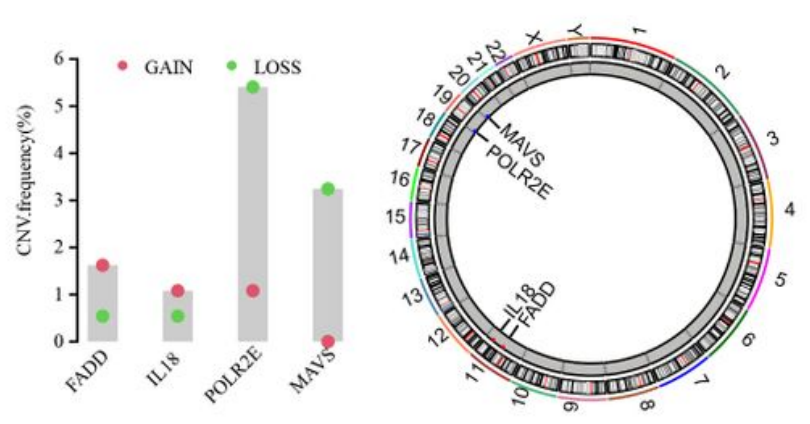

d

POLR2E

MAVS
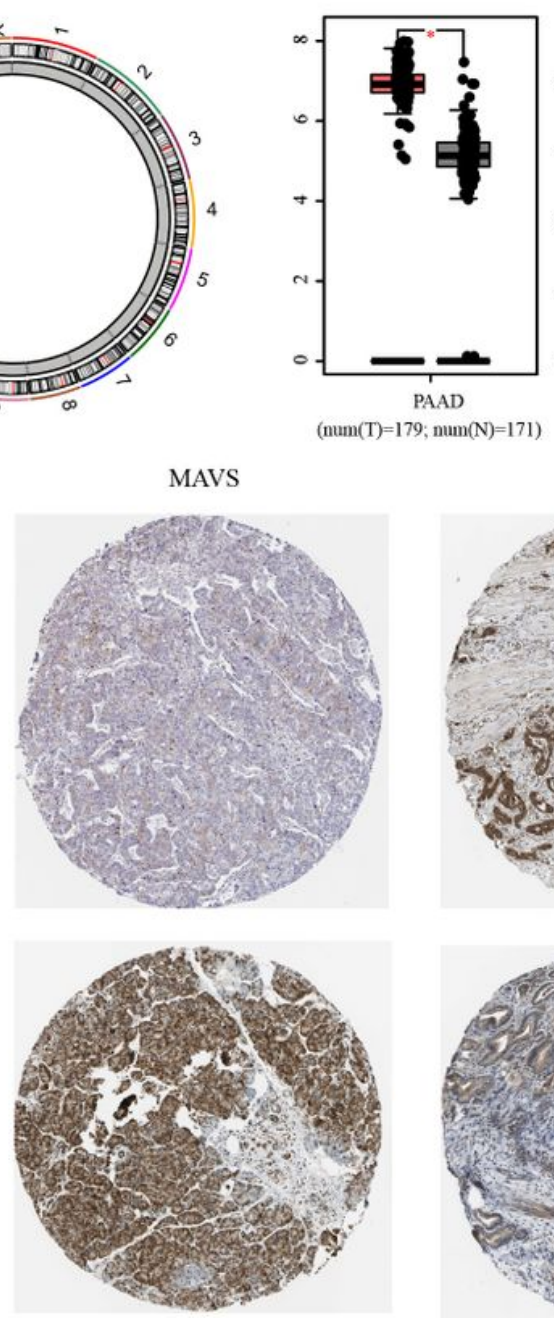

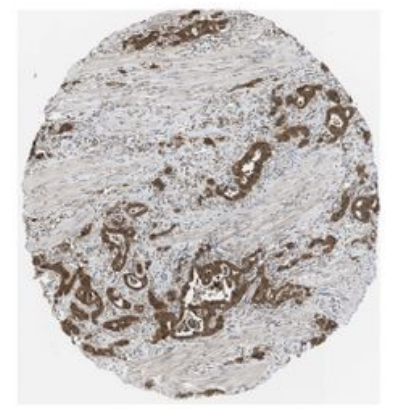

FADD

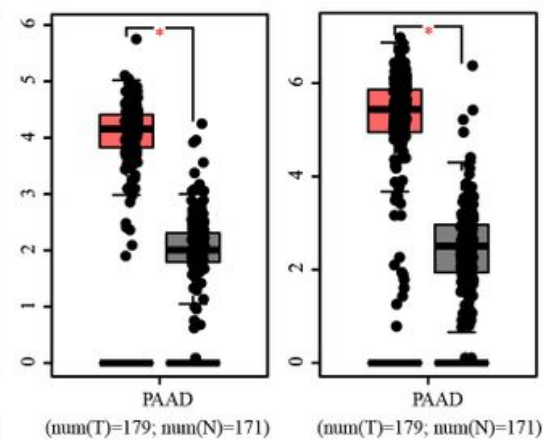

IL18
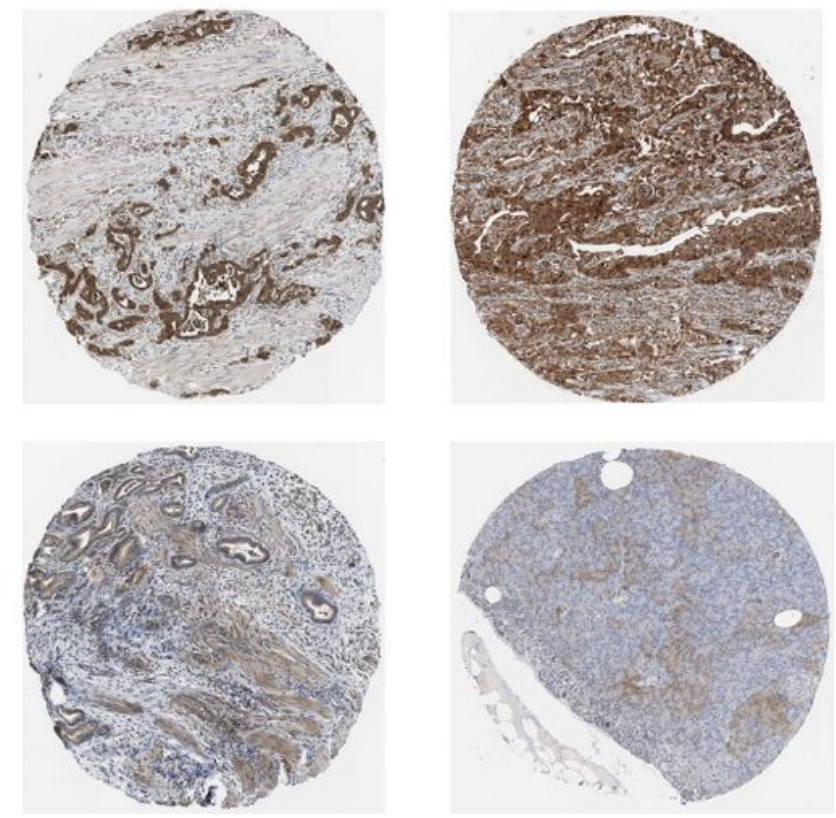

Figure 7

The landscape of genetic alterations of four signature genes in PC. a The CNV mutation frequency of four signature genes was prevalent. $b$ The location of CNV alteration of four signature genes on chromosomes. $c$ The difference of mRNA expression of four signature genes base on GEPIA database. $d$ The protein expression levels of four signature genes based on the HPA database. 
a

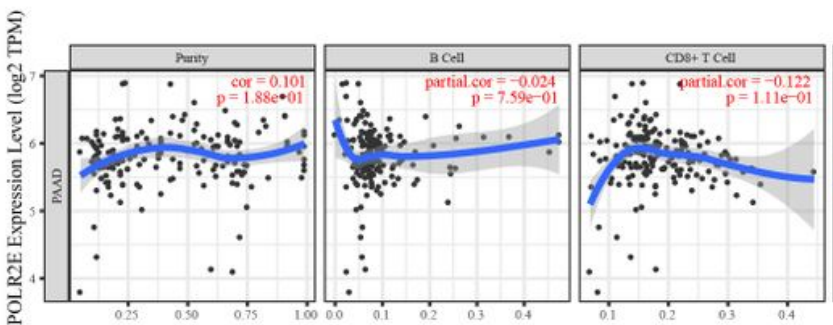

b

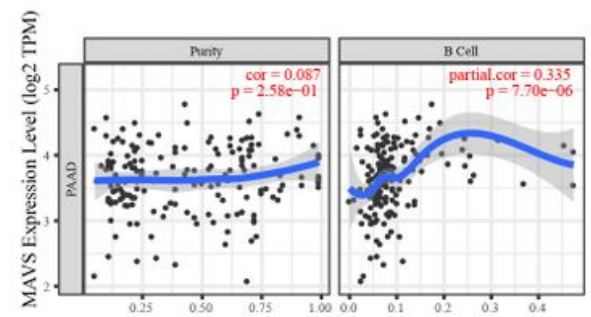

C

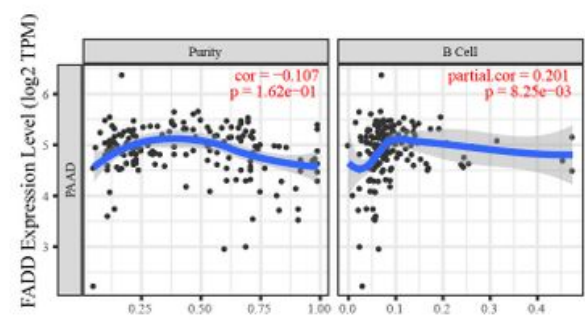

d
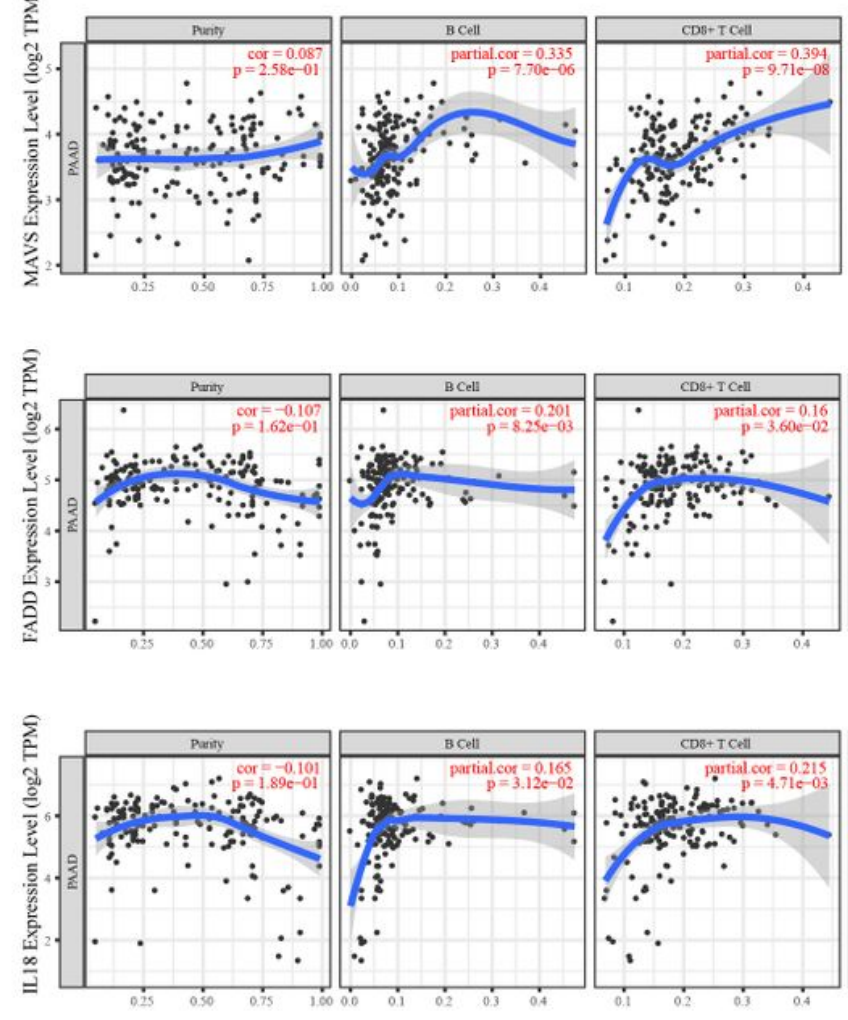
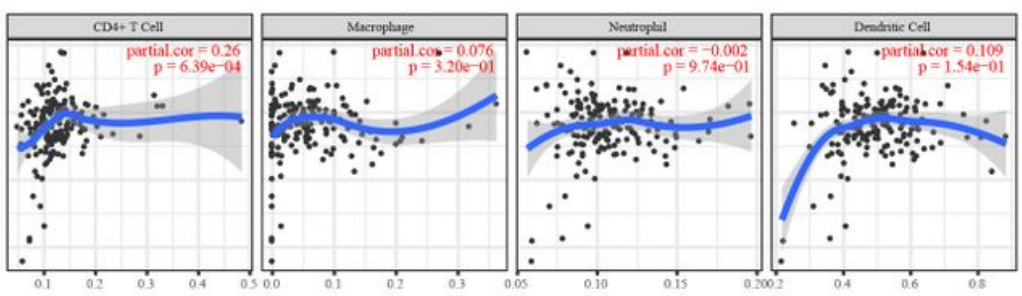

Infiltration Level
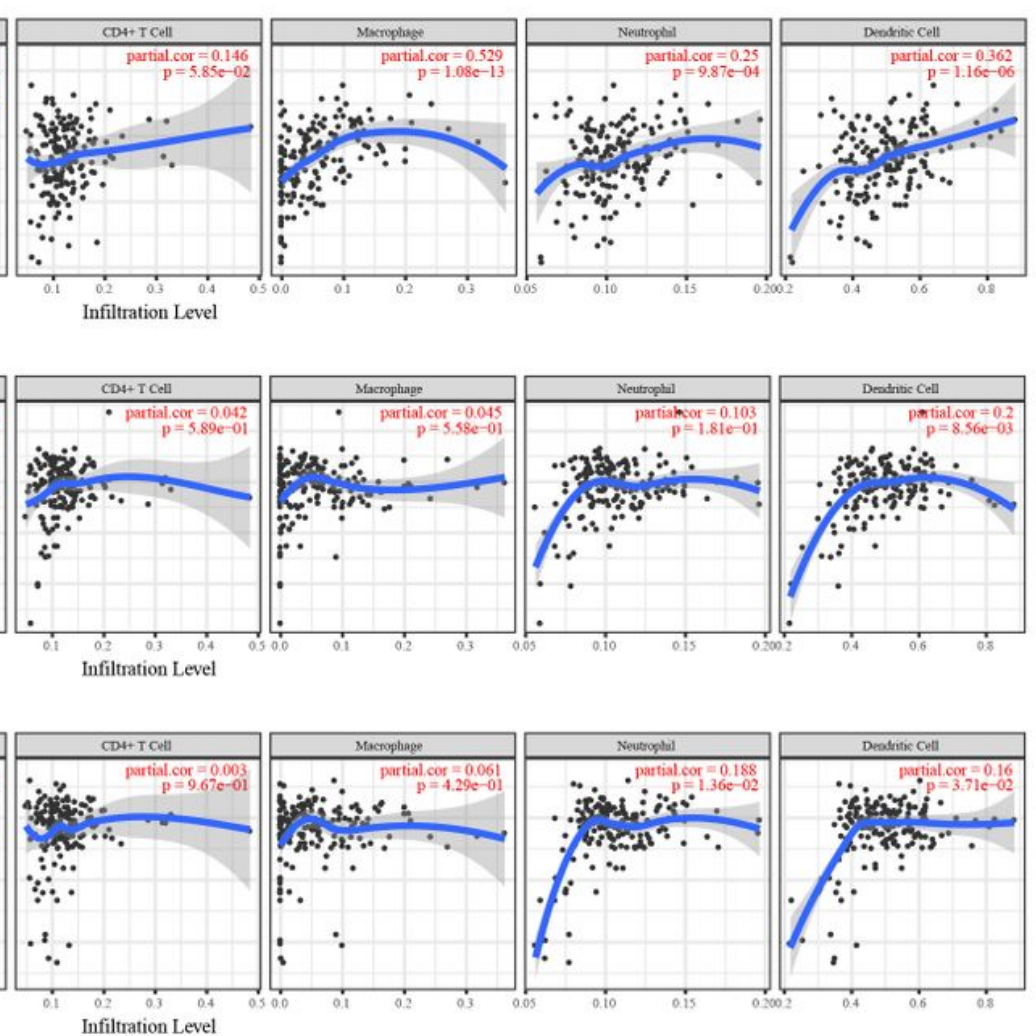

Figure 8

Relationships between the four signature genes and the infiltrating levels of six immune cells in PC. a POLR2E, b MAVS, c FADD, and d IL18.
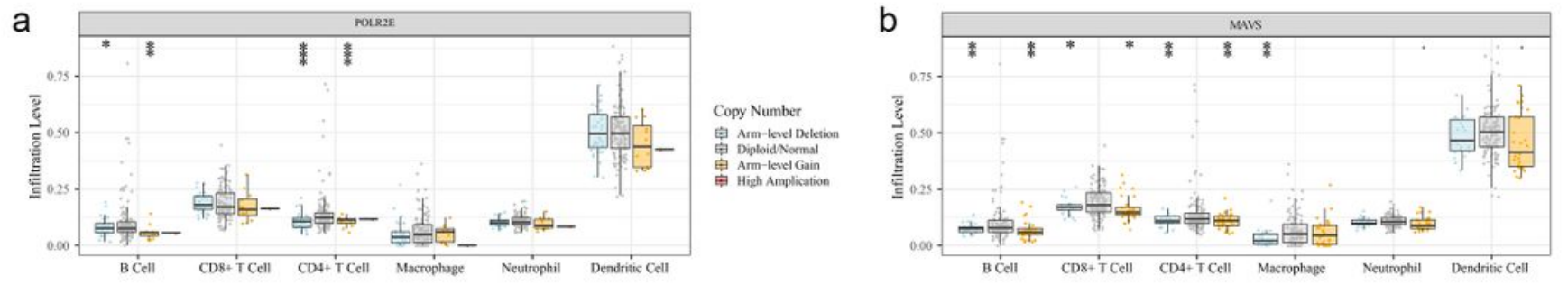

Copy Number Arm-kevel Deletion
Diploid Normal Arm-level Gain
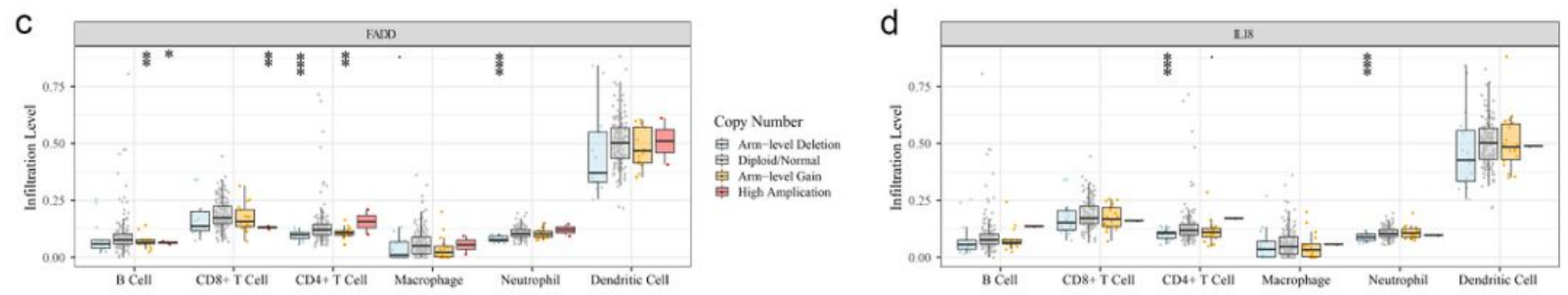

Copy Number

帛 Arm-level Deletio ir Arm-lovel Gain 角 High Amplication 
Figure 9

Effect of the Genetic Alterations of four signature genes on the Immune Cell Infiltration. a POLR2E, b MAVS, c FADD, and d IL18. *P $<0.05$.
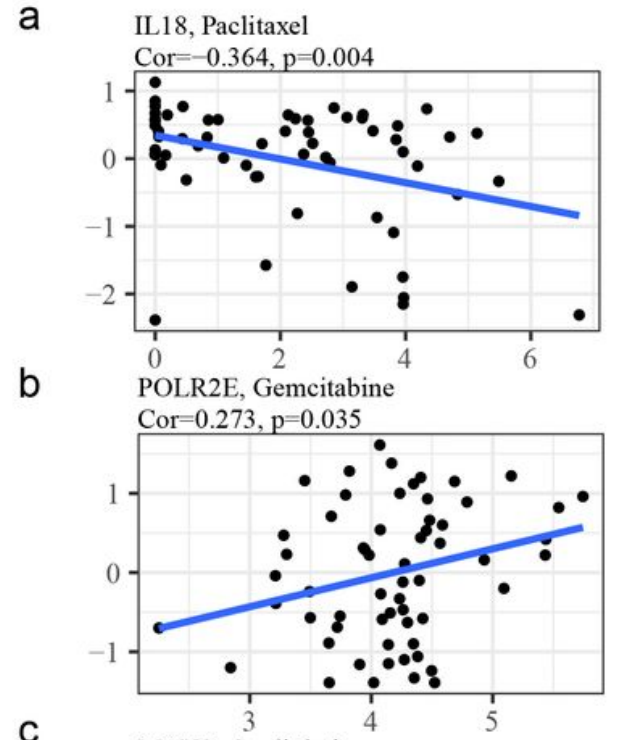

C

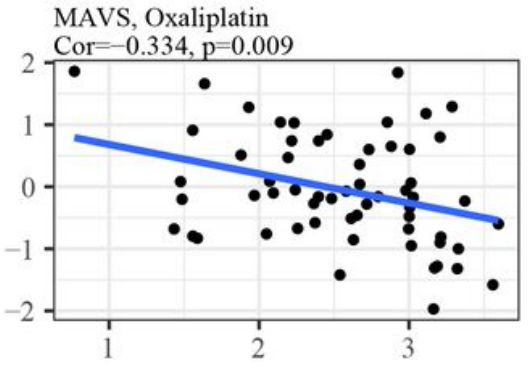

d

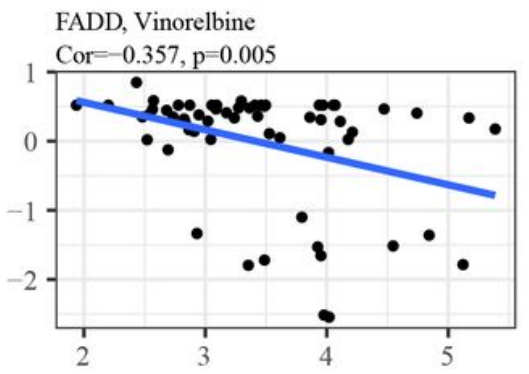

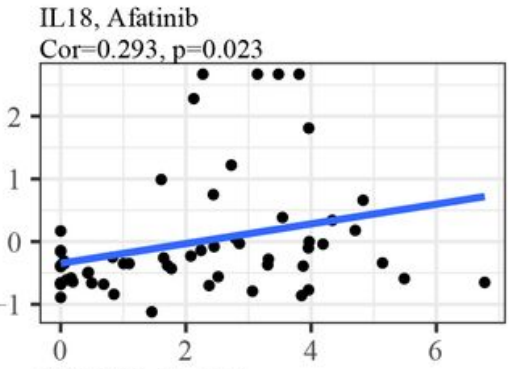

POLR2E, Cisplatin

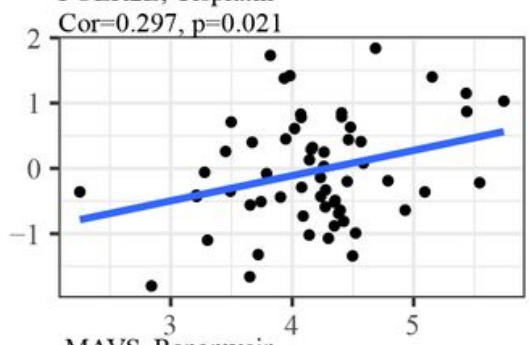

MAVS, Rapamycin

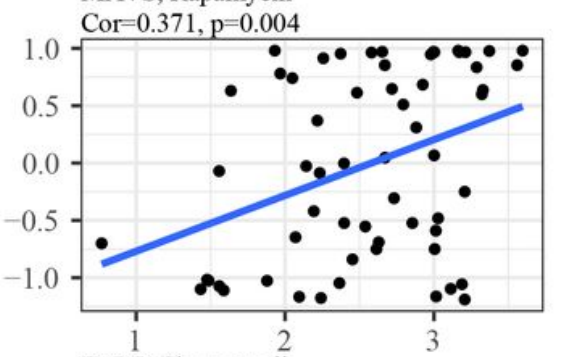

FADD, Fluorouracil

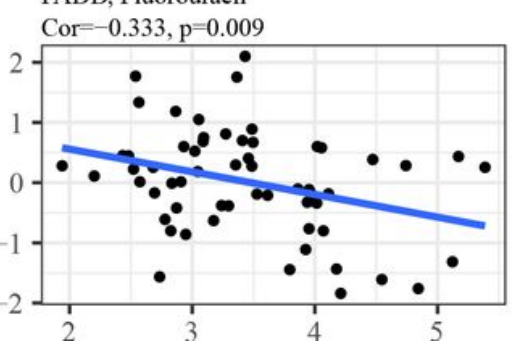

IL18, Hypothemycin

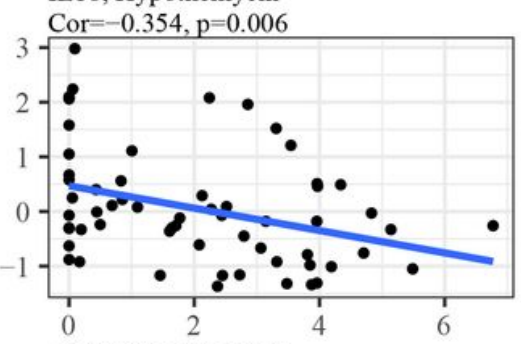

POLR2E, Carboplatin

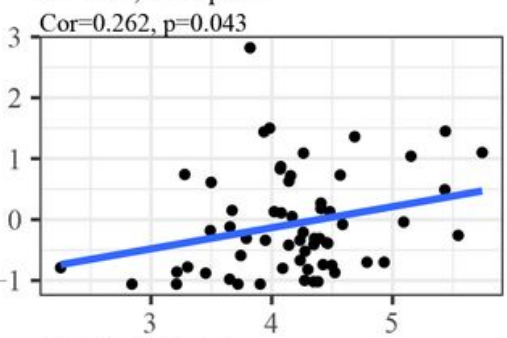

MAVS, Paclitaxel

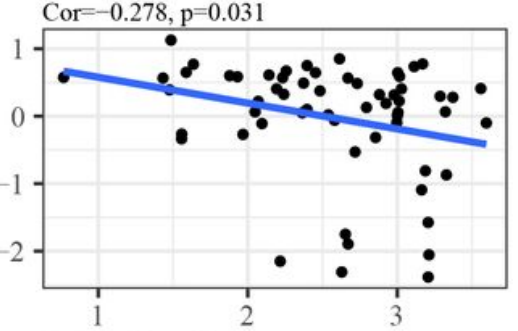

FADD, Tamoxifen

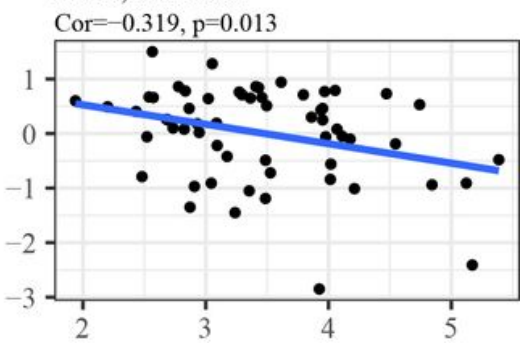

Figure 10

Drug sensitivity analysis of four signature genes. a IL18, b FADD, c MAVS, and d POLR2E.

a

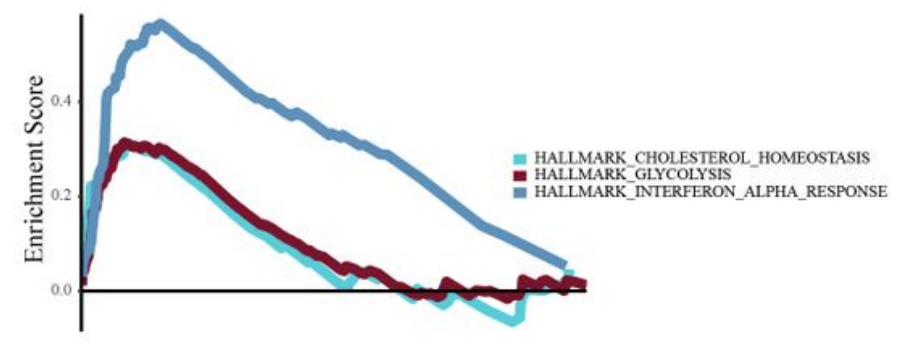

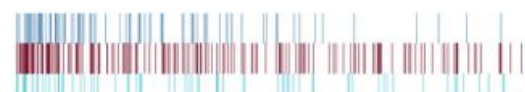

high risk $<-\longrightarrow$ - $>$ low risk b
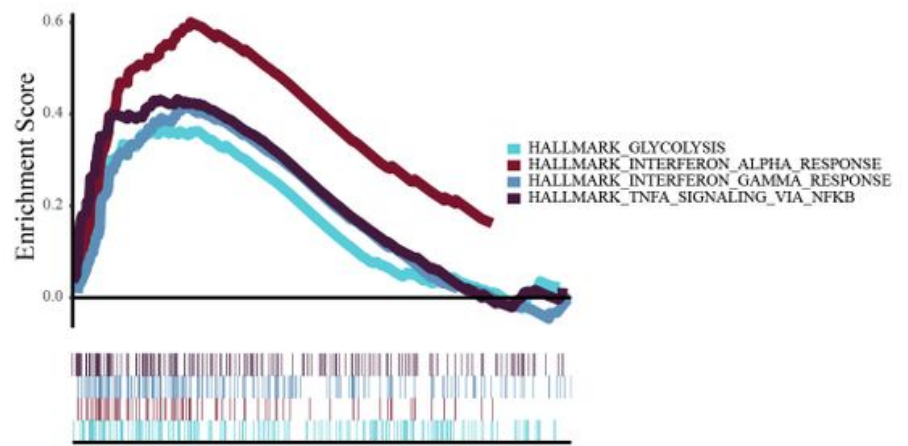

high risk $<-\longrightarrow+>$ low risk 


\section{Figure 11}

Gene set enrichment analysis of risk scores associated with signaling pathways hallmark datasets. a TCGA database. b GEO database.

\section{Supplementary Files}

This is a list of supplementary files associated with this preprint. Click to download.

- SupplementaryTable1.docx 